\title{
Dynamic Mathematical Model of Information Spreading on News Platform
}

\author{
Guohui Song $\mathbb{D}^{1}{ }^{1}$ Yongbin Wang $\mathbb{D}^{1},{ }^{1}$ and Yugang $\mathrm{Li}^{2}$ \\ ${ }^{1}$ State Key Laboratory of Media Convergence and Communication, Communication University of China, Beijing 100024, China \\ ${ }^{2}$ Academy of Broadcasting Science, Beijing 100866, China \\ Correspondence should be addressed to Yongbin Wang; ybwang@cuc.edu.cn
}

Received 1 September 2021; Revised 8 October 2021; Accepted 21 October 2021; Published 8 November 2021

Academic Editor: Yun Tie

Copyright (C) 2021 Guohui Song et al. This is an open access article distributed under the Creative Commons Attribution License, which permits unrestricted use, distribution, and reproduction in any medium, provided the original work is properly cited.

\begin{abstract}
In recent years, the news platform has become the primary source of information for users. However, there are few studies on the news platform, especially for the analysis and modeling of the spreading process of information. This article models the dynamic process of information spreading on the news platform. Firstly, we analyze the dynamic characteristics of user state and information value. Users of news platforms have two states, active and silent states, and users can switch between these two states. The information value determines the probability of user state conversion. We construct the mathematical model for the dynamic features of user state and information value considering these characteristics. Then, with appropriate parameter assumptions, simulation experiments are performed to analyze the regularity of information spreading. The results of the experiment show that the user's reading speed $1 / r$ and the conversion probability $p(\alpha, \beta, t)$ are important indicators that affect user state conversion. The lower reading speed and higher conversion probability can improve the transformation of the user state. Furthermore, we present some applications to promote information spreading, such as assessing the effectiveness of information spreading and controlling rumors on news platforms. Finally, we analyzed the effect of its information dissemination by taking Toutiao as an example and confirmed that the visibility and quality of information are important factors that affect information spreading. The experiments and analysis show that the dynamic mathematical model can reflect the information spreading in different situations with different parameters on the news platform.
\end{abstract}

\section{Introduction}

At present, the news platform has become the essential way for users to get information. In China, some news platforms represented by Toutiao and Tencent News rely on big data technology and recommendation algorithms to increase user stickiness by meeting users' personalized demands, making them a media platform with strong competitiveness. At the end of 2020, the mobile news platform recorded monthly active users of more than 600 million, and the penetration rate of the entire network is close to $80 \%$. Among them, the top platforms such as Toutiao and Tencent News maintain their dominant positions and reach the level of billions MAU [1] (Figure 1). These platforms play an important role in the spread of information between users and platforms. Therefore, understanding the mechanism of information spreading can facilitate assessing the effectiveness of information spreading and the design of information pushing strategies on the news platform.

In recent years, studying the dynamic process of information spreading has become a challenging task because it can help people understand the mechanism of information spreading [2]. Among them, the most common methods are to observe the flow of information by tracking the amount of information spread on the social network. Most studies focus on rumor control [3-5], traceability of fake news [6-8], avoiding economic crises [9], and improving the effectiveness of information spreading $[10,11]$. Due to the enormous value in the political and economic fields and the demand for commercial engineering applications, increasing attention has been paid to information spreading $[12,13]$. Meanwhile, the above research focuses mainly on 


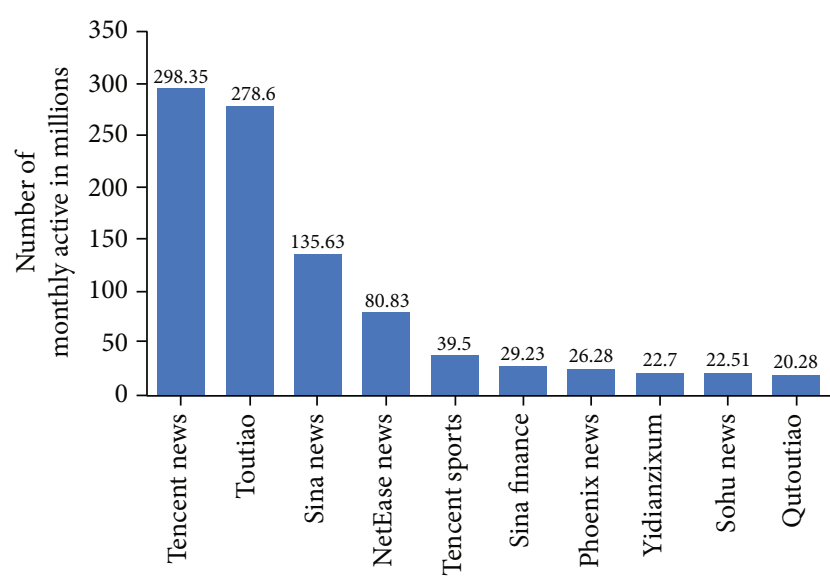

FIGURE 1: Number of monthly active users (MAU) of the leading news platform in China in December 2020.

social networks. The theory of epidemic spreading [14-17] and the complex network model [18-21] can be applied to information spreading on the social network. In addition, with the rapid development of the $5 \mathrm{G}$ mobile Internet, user mobility prediction $[22,23]$ based on mobile network access also has an important impact on the information spreading on the social network. The above research focuses mainly on social networks, and the main idea is to use differential equations to model dynamic changes in user states. Although the research objects are different, the idea of modeling inspires our work.

Through the investigation of existing researches, the current works on information spreading are mainly applied to social networks, such as Twitter and Facebook. Although some news platforms have become the primary sources of information for users, few studies focus on them, especially for analyzing and modeling the spreading process of information on news platforms.

Unlike social networks, information spreading on news platforms mainly relies on some factors such as information content and timeliness. News platforms such as Toutiao and Tencent News are not information producers themselves. They collect news from the Internet through crawler technology and use personalized recommendation technology to push information. However, most news platforms serve only as a medium for information spreading and have minor social attributes compared to social network platforms such as Weibo or Twitter. From a global perspective, the news platform pushes a large amount of information every day. The push of the information from the platform, information consumption, and the demise of the information is a dynamic process. The dynamic characteristics of information on news platforms include two main aspects: (1) The dynamic characteristics of the user state: the users on news platforms are consumers of information. At a particular moment $t$, the user's state may be active or silent, which means whether the user is reading the information. The user's state will change from silent to active according to a particular probability $p(\alpha, \beta, t)$ due to the information value. Based on the dynamic characteristics of users, the informa- tion spreading on news platforms means that users turn from the silent state to the active state. (2) The dynamic characteristics of information: the value of information on news platforms changes dynamically, and changes in information value are related to some factors such as the quantity value of information, visibility value of information, and the time $t$. Because the information pushed by news platforms is diverse, it should be noted that we are concerned with the value of information on the same topic. The value of information determines the possibility of the user's transformation from a silent state to an active state. The greater the value of information, the easier it is for silent users to convert and the more helpful it is to spread information. Therefore, the mathematical model of the user state and information value allows us to study further information spreading on news platforms.

Based on the dynamic characteristics of information spreading on news platforms, we can construct a mathematical model to describe news platforms' dynamic information spreading process. This paper tries to answer the following two questions: (1) On the news platform, how does the user's state change dynamically? What guidance do dynamic changes of user state have on news platform information push? (2) How does the value of information on news platforms change? How do we quantitatively represent the changing process of information spreading?

The remainder of this paper is organized as follows. We introduce the dynamic characteristics of information spreading on news platforms in Section 2. In Section 3, we construct the dynamic mathematical model based on the dynamic characteristics of user state and information value. In Section 4, the numerical simulation method and parameter settings are introduced. In Section 5, we introduce some applications based on the dynamic mathematical model. Meanwhile, we take Toutiao as a research object to verify the influence of model parameters on the effect of information spreading. In Section 6, conclusions are presented.

\section{Dynamic Characteristics during Information Spreading on News Platform}

In this section, we first introduce the dynamic characteristic of the user state. Then, the dynamic characteristics of the information are presented. Analyzing these characteristics can help understand the mechanism of information spreading on the news platform and provide a basis for constructing a dynamic mathematical model.

2.1. Dynamic Characteristics of the User State. In the process of information spreading on the news platform, the subjects are users whose state triggers the spreading of information. Dynamic is a typical feature of the user on news platforms, and the user state determines whether the information is effectively spread. At a particular moment $t$, some users may be in the active state. At the same time, the other users may be silent. The state of the users is transformed according to a certain probability. Some factors determine the probability, and the following factors are closely linked. 
(1) Quantity value of information $\alpha$ indicates the attractiveness of information to silent users. The more the quantity value, meaning people are more willing to read, the higher the conversion probability

(2) Visibility value of information $\beta$. The visibility of information depends mainly on the push method of the news platform, such as personalized recommendations. The higher the visibility of the information, the higher the conversion probability

(3) Time value of information appearing $t$ indicates the freshness of information. The more it means that the information is outdated and less attracted, the lower the conversion probability. The conversion probability can be denoted by $p(\alpha, \beta, t)$ considering the dynamic characteristics of the user state. The quantity value $\alpha$ and the visibility value $\beta$ are determined only by the information itself. Thus, $\alpha$ and $\beta$ are ascertained once the information emerges. We define $\alpha$ and $\beta$ as the constant that will not change in the process of information spreading. Thus, in the function of $p(\alpha, \beta, t)$, the parameter $t$ is only a variable. Therefore, we use $p(t)$ instead of $p(\alpha, \beta, t)$ in the following part. Since we consider the overall dynamic characteristics of news platforms, the value of $p(t)$ describes the average probability of user state conversion

Based on the above analysis, we can make the following definition.

(1) At any moment $t$, the probability that the users transfer from the silent state to the active state was denoted as $p(t) \in(0,1]$

(2) Suppose that the average speed for the users to read information is $1 / r$. After reading a piece of information, the users will change from the active state to the silent state at a speed of $1 / r . r$ is the average time for users to read information. Through the investigation of current research, some studies indicate that the user's reading speed is related to the length of information content, the user satisfaction, the user's historical average reading speed, and the reading dwell time $[24,25]$. Some scholars have also studied the effects of mobile device display (such as fonts and line spacing) on reading speed $[26,27]$. Therefore, the factors that affect reading speed are complex. In this paper, we set $1 / r$ as a constant, representing the average reading speed of all users

Based on the above assumptions, we can describe the dynamic characteristics of the user state as follows: at any moment $t$, users in the silent state can convert into the active state with a probability of $p(t)$. Meanwhile, users in the active state will turn into the silent state at a speed of $1 / r$. It will implement effective information spreading once silent users become active users.

2.2. Dynamic Characteristics of Information Value. The information value on news platforms also has dynamic char- acteristics. The dynamic characteristic of information value refers to the fact that the attention of information content will change over time. Users' attention to a particular topic is a decisive factor in determining the effectiveness of information dissemination. From a global perspective, at any moment $t$, we assume that the average value of information on a particular topic is determined by the push speed of information and the total amount of information about the topic. The following factors are closely linked.

(1) The value $L$ indicates the life cycle of the information

(2) At any moment $t$, for information about a particular topic, the total amount of information transmitted by the news platform is defined as $w(t)$. Because of the limitation of the information push capability of the news platform, we assume that the maximum amount of information is $R$ in the life cycle of the information. So, the value of $w(t)$ is between 0 and $R$. It means that at the initial moment $t=0$, the news platform does not push information. At the moment $t=L$, the maximum amount of information transmitted by the news platform is $R$

(3) At any moment $t$, the information push speed of the news platform is expressed as $w^{\prime}(t)$

(4) In the information life cycle $L$, the more information-pushed before $t$ moment, the easier it is for information-pushed after $t$ moment to lose attention for a particular topic. At any moment $t$, the mean value of the information value of the news platform is expressed as $v\left(w^{\prime}, w\right)$. The faster the topic information is updated, the easier it is for the users to pay attention to a particular topic. However, the more information on this topic, the easier it is to lose interest in the users. For simplicity, we assume that $v\left(w^{\prime}, w\right)=w^{\prime}(t) / w(t)$, it means that the average information value is directly with $w^{\prime}(t)$ and inversely with $w(t)$

\section{Dynamic Mathematical Model of Information Spreading}

From the above analysis of the dynamic characteristics of the news platform, we can consider the effect of information spreading on news platforms from different perspectives. Based on the dynamic characteristics of the user state, we can analyze the number of active users at a particular moment $t$. Based on the dynamic characteristics of information value, we can analyze the changes of information value of a particular topic during the information life cycle.

3.1. Modeling for Dynamic Characteristics of User State. In the process of information spreading, users of news platforms have two states: the active state and the silent state. At any moment $t$, users who are in the silent state do not get information from the news platform, and they can convert to the active state with a probability of $p(t)$. Meanwhile, the users in the active state will turn into the silent state at a 
speed of $1 / r$. It will implement effective information spreading once the silent users become active users. The conversion process of the user state is shown in Figure 2.

Based on the above rules, we can make the following assumptions and definitions for the model parameters.

(1) The total number of users on the news platform is defined as $n$, and the information is sufficient

(2) At moment $t$, the active and silent users on the news platform are $g(t)$ and $f(t)$, respectively. The $g(t)$ and $f(t)$ meet the following equation

$$
f(t)+g(t)=n \text {. }
$$

(3) Every piece of information on the news platform has the same life cycle, which is defined as $L$

(4) At the moment $t$, the rate of change of the user from the silent state to the active state can be expressed as $f(t) \times p(t)$. The $p(t)$ is the probability that users transition from the silent state to the active state

The dynamic model can be constructed according to the user state's dynamic characteristics and the information value's dynamic characteristics. Suppose that the number of users in the active state at the moment $t$ is $g(t)$ and the number of users in the silent state is $f(t)$, the keynote of the dynamic characteristics of users can be presented in the following formula:

$$
g(t)+f(t) \stackrel{p(t), 1 / r}{\longrightarrow} g(t+\Delta t)+f(t+\Delta t)
$$

The above formula expresses the dynamic change process of the user state when the total number of users is constant $n$. This process is mainly affected by conversion probability $p(t)$ and reading speed $1 / r$. At the moment $t+$ $\Delta t$, the dynamic change of the number of users in the active state can be expressed by the following formula:

$$
g(t+\Delta t)=g(t)+f(t) p(t) \Delta t-\frac{g(t)}{r} \Delta t
$$

From the moment $t$ to $t+\Delta t$, the change process of the number of users in the active state can be described as follows. (1) The silent state users turn into the active state with probability $p(t)$. (2) The users in the active state turn into the silent state after reading some information at a speed of $1 / r$. Therefore, the rate of change of active users can be calculated by the following formula:

$$
\begin{aligned}
\frac{d g(t)}{d t} & =\lim _{\Delta t \rightarrow 0} \frac{(g(t)+f(t) p(t) \Delta t-(g(t) / r) \Delta t)}{\Delta t} \\
& =f(t) p(t)-\frac{g(t)}{r}=(n-g(t)) p(t)-\frac{g(t)}{r} .
\end{aligned}
$$

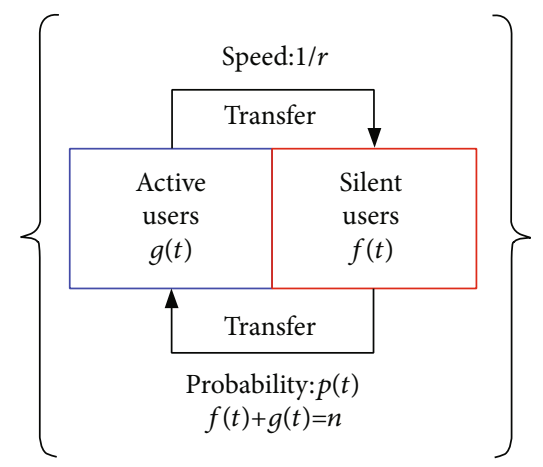

FIGURE 2: The dynamic conversion process of user state on news platform.

Through the above transformation, the change rate of the active users can be obtained by solving the following first-order linear differential equation:

$$
\frac{d g(t)}{d t}=-\left(p(t)+\frac{1}{r}\right) g(t)+n p(t)
$$

Solving the first-order linear differential equation generally uses the method of constant variation, and this method can obtain the solution of the first-order linear differential equation. First, we can solve the corresponding linear equation, and the linear equation is as follows:

$$
\frac{d g(t)}{g(t)}=-\left(p(t)+\frac{1}{r}\right) d t
$$

Then, we can transform the above formula:

$$
\ln g(t)=-\int\left(p(t)+\frac{1}{r}\right) d t
$$

Finally, we can get the general solution of this homogeneous equation; the $C$ is constant and it is determined by the initial conditions of the function. Therefore, the number of users in the active state $g(t)$ at the moment $t$ can be represented as

$$
g(t)=C e^{-\int(p(t)+1 / r) d t}
$$

Let $C=c(t)$; the above general solution of $g(t)$ can be expressed as

$$
g(t)=c(t) e^{-\int(p(t)+1 / r) d t}
$$

We further process the above formula:

$$
\frac{d g(t)}{d t}=\frac{d c(t)}{d t} e^{-\int(p(t)+1 / r) d t}-c(t)\left(p(t)+\frac{1}{r}\right) e^{-\int(p(t)+1 / r) d t}
$$

After inserting equations (5) and (9) into equation (10), we can obtain 


$$
\begin{gathered}
\frac{d c(t)}{d t} e^{-\int(p(t)+1 / r) d t}-c(t)\left(p(t)+\frac{1}{r}\right) e^{-\int(p(t)+1 / r) d t} \\
=-\left(p(t)+\frac{1}{r}\right) c(t) e^{-\int(p(t)+1 / r) d t}+n p(t)
\end{gathered}
$$

We further deduce the above equation (11):

$$
\begin{aligned}
\frac{d c(t)}{d t} & =n p(t) e^{\int(p(t)+1 / r) d t} d t, \\
c(t) & =\int n p(t) e^{\int(p(t)+1 / r) d t} d t+\bar{c} .
\end{aligned}
$$

After inserting equation (13) into equation (9), the general solution of $g(t)$ can be described as the following formula:

$$
g(t)=e^{-\int(p(t)+1 / r) d t}\left(\int n p(t) e^{\int(p(t)+1 / r) d t}+\bar{c}\right) .
$$

The value of $\bar{c}$ is determined by the initial conditions of the model. Therefore, according to equations (1) and (14), the number of silent users $f(t)$ at the moment $t$ can be expressed as

$$
f(t)=n-e^{-\int(p(t)+1 / r) d t}\left(\int n p(t) e^{\int(p(t)+1 / r) d t}+\bar{c}\right) .
$$

Suppose that at the moment $t=0$, the number of active users $g(t)=0$. In other words, at the initial moment of the model, the information on the news platform has not been disseminated. It is worth noting that at the moment $t=0$, $p(t) \neq 0$, because at the initial moment of the model, there is already information on the news platform for users to read. Through the above analysis, we can obtain the value of $\bar{c}$.

$$
\begin{gathered}
e^{-\int(p(t)+1 / r) d t}\left(\int n p(t) e^{\int(p(t)+1 / r) d t}+\bar{c}\right)=0, \\
\bar{c}=-\int n p(t) e^{\int(p(t)+1 / r) d t} .
\end{gathered}
$$

Through the dynamic characteristics of the user state, we can construct the dynamic mathematical model to describe the process of information spreading, and at the same time, we can get a general representation of the number of active users $g(t)$ and silent users $f(t)$. With a certain number of news platform users, we can find that reading speed $1 / r$ and conversion probability $p(t)$ are factors that affect $g(t)$. The conversion probability $p(t)$ is determined by information value. The number of active users at the moment $t$ can be used to measure the effect of information spreading on the news platform.

3.2. Modeling for Dynamic Characteristics of Information Value. To further study information spreading based on the dynamic characteristics of information value, the following dynamic function is assumed:

$$
v\left(w^{\prime}, w\right)=\frac{w^{\prime}(t)}{w(t)}
$$

The function can reflect a characteristic of information value dynamics with time. At the moment $t$, the mean value of the information about a particular topic on the news platform is expressed as $v\left(w^{\prime}, w\right)$. Therefore, the total value of information from the initial time to $t$ moment can be expressed as

$$
\int_{0}^{t} \mathrm{v}\left(\mathrm{w}^{\prime}, \mathrm{w}\right) \mathrm{w}^{\prime}(\mathrm{t}) d t
$$

Equation (19) means that the total information value of a particular topic is determined by the speed of information push speed $w^{\prime}(t)$ and the total amount of information pushed by the news platform $w(t)$. One of the purposes of news platforms to push information is to maximize the value of information during the information life cycle. Then, the total value of information of a particular topic can be calculated by the following variational equation:

$$
\left\{\begin{array}{l}
\max \int_{0}^{L} \mathrm{v}\left(\mathrm{w}^{\prime}, \mathrm{w}\right) \mathrm{w}^{\prime}(\mathrm{t}) d t \\
w(0)=0, w(L)=R .
\end{array}\right.
$$

The value of $w(t)$ is between 0 and $R$. The $L$ represents the life cycle of information. For a convenient expression, we rewrite $F\left(w^{\prime}, w\right)=w^{\prime}(t)^{2} / w(t)$. According to the variational method of extreme functional value and the famous Eulerian equation, the above variational equation can be transformed to solve the following problems:

$$
F_{w}-w^{\prime} F_{w^{\prime} w}-w^{\prime \prime} F_{w^{\prime} w}=0
$$

Solving the above Euler equations, the following solution process can be obtained:

$$
\begin{aligned}
\frac{d\left(F-w^{\prime} F_{w}\right)}{d t} & =0 \text { or } F-w^{\prime} F_{w}=c, \\
w^{\prime}(t)^{2} & =c w(t), \\
\frac{d w}{\sqrt{w(t)}} & =c d t, \\
w(t) & =\left(c_{1} t+c_{2}\right)^{2} .
\end{aligned}
$$

According to the initial condition $w(0)=0, w(L)=R$, the value of $c_{1}$ and $c_{2}$ can be obtained as follows:

$$
c_{2}=0, c_{1}=\frac{\sqrt{R}}{L} .
$$

Therefore, combing equations (25) and (26), we can obtain 


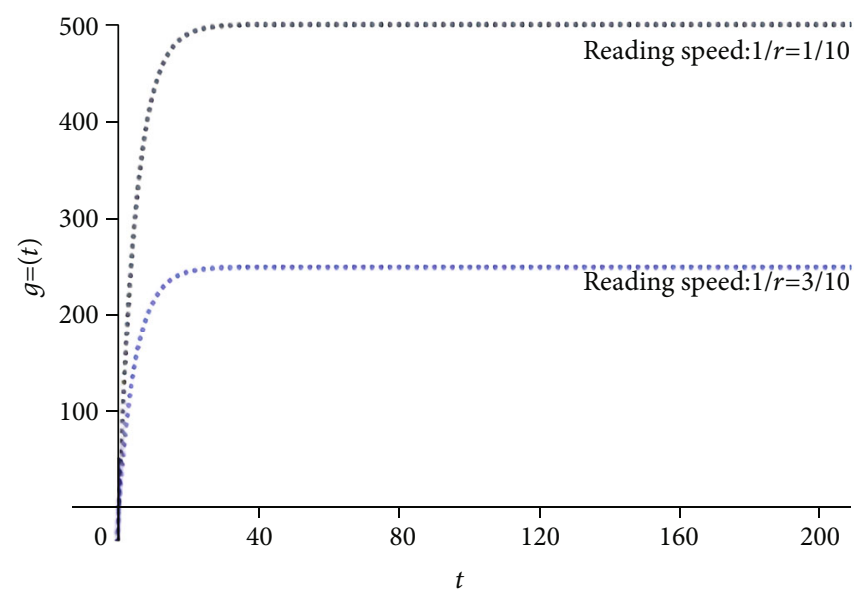

FIgURE 3: Simulation results of active users when reading speed $1 / r=1 / 10$ or $3 / 10$ and $p(t) \equiv 1 / 10$.

$$
w(t)=\frac{R t^{2}}{L^{2}} .
$$

After inserting $w(t)$ into equations (18), we thus obtain the information value at the moment $t$ :

$$
v=\frac{w^{\prime}(t)}{w(t)}=\frac{2}{t}
$$

From the above derivation, the value of information on news platforms is closely related to time. Through equation (28), we can quantitatively express the relationship between time and information value. The value of information determines the conversion probability $p(t)$. In this section, we only consider the impact of time on the value of information. In the experiment, we will consider the impact of information quality and information visibility on the value of information.

\section{Parameter Assumption and Simulation Experiment}

To further study information spreading based on the dynamic model, we can make assumptions about the model parameters. From the definition above, at the moment $t=0$, the number of active users $g(t)=0, p(t) \neq 0$. If we set the values of $p(0)$ and $1 / r$, according to equation (17), we can get the value of $\bar{c}$. Assuming $p(0)=1 / 10, n=1000,1 / r=1 /$ 10 , or $1 / r=3 / 10$, we can get $\bar{c}=-500$ or $\bar{c}=-250$. This section will discuss the impact of the three manifestations of conversion probability on information spreading.

(1) The dynamic conversion probability $p(t)$ is set to a constant. We define $p(t)$ as the initial value, that is, $p(t) \equiv 1 / 10$. The $p(t)$ is constant which means that the value of information remains stable on the news platform, resulting in the dynamic conversion probability remaining unchanged. Although this case is only a hypothetical state, it can have a particular guiding significance for the platform's information push. Then, at the moment $t$, the active users $g(t)$ can be calculated by equation (14):

$$
g(t)=e^{-c_{1}-t / 5}\left(500 e^{c_{1}+t / 5}+c_{1}-500\right) .
$$

We set $c_{1}=0$. We will still set such parameters to effectively represent the solution of the indefinite integral in the following part. Then, equation (29) can be simplified as the following formula:

$$
g(t)=500 e^{-t / 5}\left(e^{t / 5}-1\right)
$$

At the same time, to analyze the influence of user reading speed on information spreading, we set $1 / r=3 / 10$. Based on the parameters above, we can obtain the number of active users at the moment $t$ on the news platform as shown in Figure 3.

As mentioned above, $p(t) \equiv 1 / 10$ is assumed, which means the value of information is unchanged during information spreading. In this case, the dynamic characteristic of users is only considered, and simulation experiments are carried out without considering the characteristic of information value. It can be seen that the active users reach a steady state due to the dynamic characteristic of users at the moment $t$. The final value of $g(t)$ will be different according to the initial conditions. The lower the average reading speed, the faster the users will convert to an active state.

(2) The value of the conversion probability $p(t)$ is related to the dynamic characteristics of information value. Based on the above definition, we can define information value without considering other factors affecting information value by referring to equation (28). The probability of users changing from the silent state to the active state is related to time. The higher the value of the information, the easier it is for the silent users to become active users. Regardless of other factors that affect the information value, the dynamic conversion probability $p(t)$ should be 


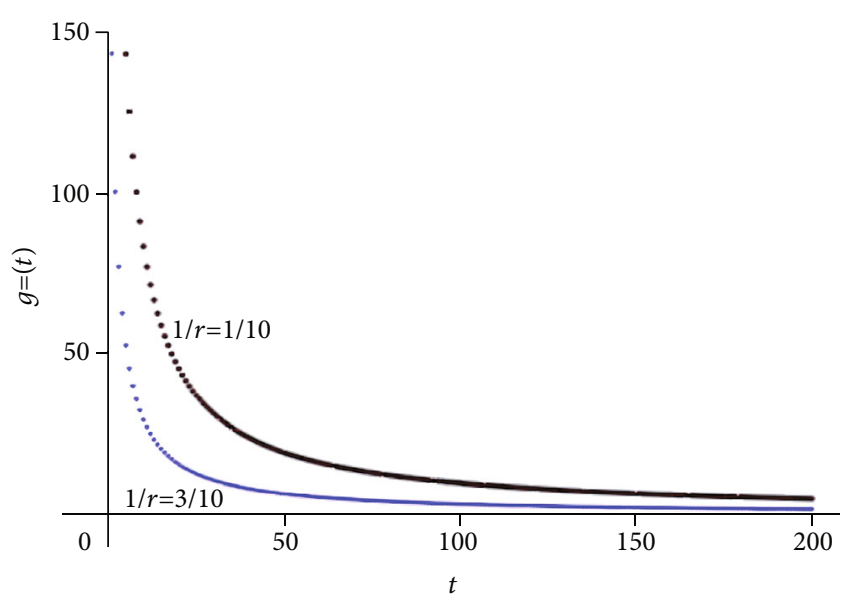

Figure 4: Simulation results of active users when $1 / r=1 / 10$ or $3 / 10$ and $p(t)=c(2 /(t+1)), c=1 / 20$.

proportional to $v$ and meet the condition $0<p(t)$ $\leq 1$, so the $p(t)$ can be defined as the following formula:

$$
p(t)=c \frac{2}{t+1}
$$

The $c$ is constant. If $c=1 / 20$, then $p(0)=1 / 10$.

Based on equations (14) and (31), assuming $n=1000$ and $1 / r=1 / 10$, the $g(t)$ can be calculated. At the same time, to analyze the influence of reading speed on information spreading, we also set $1 / r=3 / 30$. Based on the parameters above, we can obtain the number of active users on the news platform, as shown in Figure 4.

Because of the dynamic characteristics of information value, the conversion possibility will decline continuously. The variable conversion possibility is the fundamental principle of information spreading. In this case, we also consider the dynamic characteristic of the users and the dynamic characteristic of information value. The information value is due to time. As shown in Figure 4, it can be seen that the number of active users gradually decreases to a certain extent and reaches a stable value. Information value will be reduced by time because of the dynamic characteristics. It is difficult for active users to reach a high value. The lower the average reading speed, the faster the users will convert into an active state.

(3) The quantity value of information $\alpha$ and the visibility value of information $\beta$ are essential factors that influence the conversion possibility. When the quantity value of information is higher, users are more willing to read more information to increase the conversion possibility. Meanwhile, the higher the visibility of information value, the easier it is for users to get information. Furthermore, considering the visibility and quantity value of information, we can define the conversion probability as the following equation

$$
p(\alpha, \beta, t)=p(\alpha, \beta)+p(t)
$$

The exact value of $p(\alpha, \beta)$ will lie on the specific factors of information. Since the values of $\alpha$ and $\beta$ are determined from the beginning of the information, to simplify the problem, we can consider them together. Without generality, the range of $p(\alpha, \beta)$ is shown as follows:

$$
p(\alpha, \beta)=\operatorname{rand}(0,1-p(t)) .
$$

It means that it is random in its value range. The random function (33) satisfies uniform distribution. As a result, considering the visibility and quantity value of information, the conversion probability can be calculated by the following formula:

$$
p(t)=\operatorname{rand}\left(0,1-c \frac{2}{t+1}\right)+c \frac{2}{t+1}
$$

Taking the same parameters as mentioned above, combing equations (14) and (34), and simulation experiments of this case repeated 100 times, the active users $g(t)$ can be obtained as shown in Figure 5. It can be seen that the final value of $g(t)$ is very random due to the quantity value and the visibility value of information. As in the above experiment, the lower the reading speed, the easier it is to promote the conversion of the user state.

Although the value of information decays over time, due to the information value, especially considering the visibility and quantity of information, users can more easily obtain information, which helps users transform from the silent state to the active state. For example, in the experiment shown in Figure 4, if only the time utility of the value of information is considered, the number of active users will gradually decrease and ultimately remain at a low level, less than $2 \%$ of the total number of users. In the experiment shown in Figure 5, if the visibility and quantity value of information is considered, the number of active users is more than $20 \%$ of the total users most of the time. Sometimes, it can activate almost $80 \%$ of users. Meanwhile, we can find that the lower reading speed is conducive to the conversion of the user state. In order to better illustrate the problem, we randomly observe 10 time points, and the results are shown in Table 1.

\section{Analysis of Information Spreading}

The effect of information spreading depends on how the user transitions from the silent state to the active state. The conversion ability is determined by the conversion probability $p(t)$ and the reading speed $1 / r$.

First, only considering the dynamic characteristic of the user state, the conversion probability $p(t)$ is defined as a constant. In this case, the value of $g(t)$ is closely linked with the reading speed $1 / r$.

Considering the characteristic of information value, the conversion probability $p(t)$ is related to the time, and the probability continues to decrease over time. So, the value of $g(t)$ will stabilize at a lower level. The conversion 


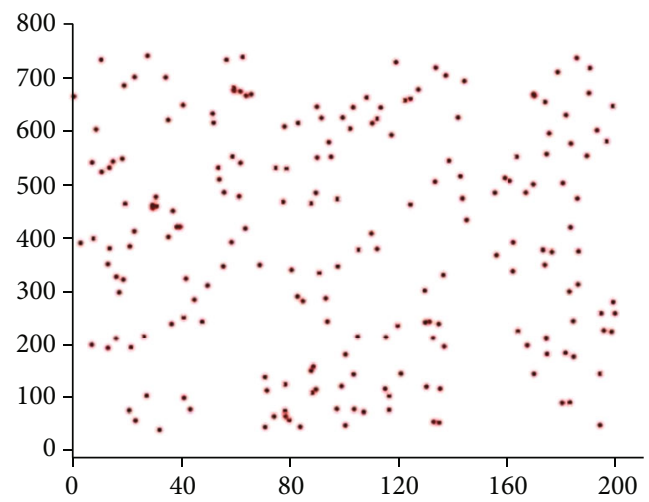

(a)

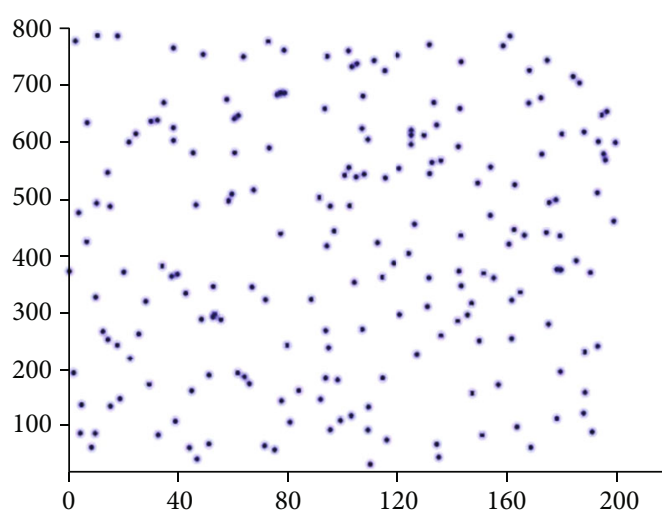

(b)

FIGURE 5: Simulation results of active users when considering the quantity and visibility value of information in the dynamic characteristics of information value: (a) $1 / r=3 / 10$; (b) $1 / r=1 / 10$.

TABLE 1: The results of information spreading with different time $t$ and reading speed $1 / \mathrm{r}$ by considering the quantity value and the visibility value of information.

\begin{tabular}{lcccccccccc}
\hline \multirow{2}{*}{ Reading speed: $1 / \mathrm{r}$} & \multicolumn{9}{c}{ Active users: $g(t)$} \\
& $g(20)$ & $g(40)$ & $g(60)$ & $g(80)$ & $g(100)$ & $g(120)$ & $g(140)$ & $g(160)$ & $g(180)$ & $g(200)$ \\
\hline $1 / 10$ & 210 & 375 & 198 & 245 & 105 & 389 & 565 & 423 & 431 & 603 \\
$3 / 10$ & 85 & 98 & 391 & 83 & 87 & 228 & 543 & 525 & 96 & 685 \\
\hline
\end{tabular}

probability should be enough so that the silent users can effectively convert to active users.

In addition to time, the conversion probability is also determined by the quantity value of information $\alpha$ and the visibility value of information $\beta$. Therefore, the conversion probability has certain degrees of randomness because the values of $\alpha$ and $\beta$ are uncertain on the news platform. Meanwhile, the number of active users is random.

In different cases with different $p(t)$, the results of information spreading can be shown in Table 2. The simulation experiments show that the active users are different due to different cases. When $p(t)$ is constant, the conversion probability will not change. Under this condition, the number of active users will reach a stable value due to the initial parameters set. When considering the relationship between the value of information and time, the conversion probability will generally decrease, so the final number of active users will reach a minimal level. Considering the value of quantity and visibility of information, the probability of conversion can change randomly, making silent users effective at some time.

With the adjustment of the reading speed $1 / r$, it can be seen that the reading speed has a particular impact on the user state conversion. The lower the reading speed, the more conducive to the user state conversion.

\section{Application and Case Study}

6.1. Application. The characteristics of user state and information value can reflect the dynamic process of information spreading on the news platform as a whole. So, we can understand the dynamic transform process of the user's state well and predict the information spreading effect according to the model mentioned above. Meanwhile, we can apply some measures to promote the information spreading.

(1) Assess the effectiveness of information spreading: on news platforms, the number of active users and the total amount of information read are critical indicators to evaluate the effect of information spreading. The total amount of information read can be described as the following formula

$$
\text { total }=\int_{0}^{L} f(t) p(t) d t
$$

According to equations (15) and (35), the total reading users of the information on the news platform can be obtained as follows:

$$
\text { total }=\int_{0}^{L}\left(n-e^{-\int(p(t)+1 / r) d t}\left(\int n p(t) e^{\int(p(t)+1 / r) d t}+\bar{c}\right)\right) p(t) d t
$$

The $L$ is the life cycle of information. The total represents the number of silent users converted into active users during the news life cycle, and it can dynamically describe the process of information spreading on the news platform. Therefore, we define total as the effect of information spreading on news platforms. During the operation of the news platform, one of the purposes of the news platform is 
TABLE 2: The results of information spreading with different conversion probability $p(t)$ and reading speed $1 / \mathrm{r}, c=1 / 20$, and $n$ $=1000$.

\begin{tabular}{lcccc}
\hline Conversion probability: $p(t)$ & Reading & \multicolumn{3}{c}{ Active users: $g(t)$} \\
& speed: $1 / \mathrm{r}$ & $g(20)$ & $g(100)$ & $g(200)$ \\
\hline $1 / 10$ & $1 / 10$ & 491 & 500 & 500 \\
\multirow{2}{*}{$2 c$} & $3 / 10$ & 245 & 250 & 250 \\
$\overline{(t+1)}$ & $1 / 10$ & 72 & 16 & 6 \\
rand $\left(0,1-\frac{2 c}{t+1}\right)+\frac{2 c}{t+1}$ & $3 / 10$ & 28 & 7 & 2 \\
& $1 / 10$ & 210 & 105 & 603 \\
\end{tabular}

to increase the reading volume of information. For this purpose, the goal of optimization is the value of total.

(2) Promote information spreading: to make silent users turn into active users as quickly as possible is an essential task on the news platform. The process of user state conversion is also the process of information spreading. The experiments and theoretical analysis show that the conversion probability $p(t)$ and reading speed $1 / r$ are essential factors in information spreading. As shown in Figures 3-5, different information spreading effects can be achieved by formulating different probability distributions. For example, suppose that the news platform needs a relatively stable information spreading effect. In that case, as shown in Figure 3, the quantity and visibility value of the information must be dynamically adjusted so that the conversion probability maintains a relatively stable value. If the news platform needs to improve the information spreading at specific time points, the visibility and quantity of information should be adjusted. Meanwhile, information spreading can be restrained by reducing the value of information in some areas, such as rumor control. The news platform can take effective measures by revealing malicious rumor information and reducing the visibility of this type of information. The value of the incorrect information or rumor will be decreased in a straight line so that the value of $p(\alpha, \beta)$ is 0 . Then, the value of information will decrease progressively so that the dissemination of rumor information will end naturally, as shown in Figure 4

6.2. A Case Study of Toutiao. Toutiao is a popular mobile news platform in China. In order to verify the influence of model parameters on the effect of information spreading, we take Toutiao as the object for further research. At present, Toutiao promotes the dissemination of mainstream value information by placing 2-3 pieces of mainstream value information on the top of the homepage. We got the two pieces of information that Toutiao put on top and analyzed the effect of its information spreading. We use the number of comments as an indicator to evaluate the user activation state. We take a crawl every 15 minutes to obtain a total of 1624 valid data and draw a scatter chart of the data in time

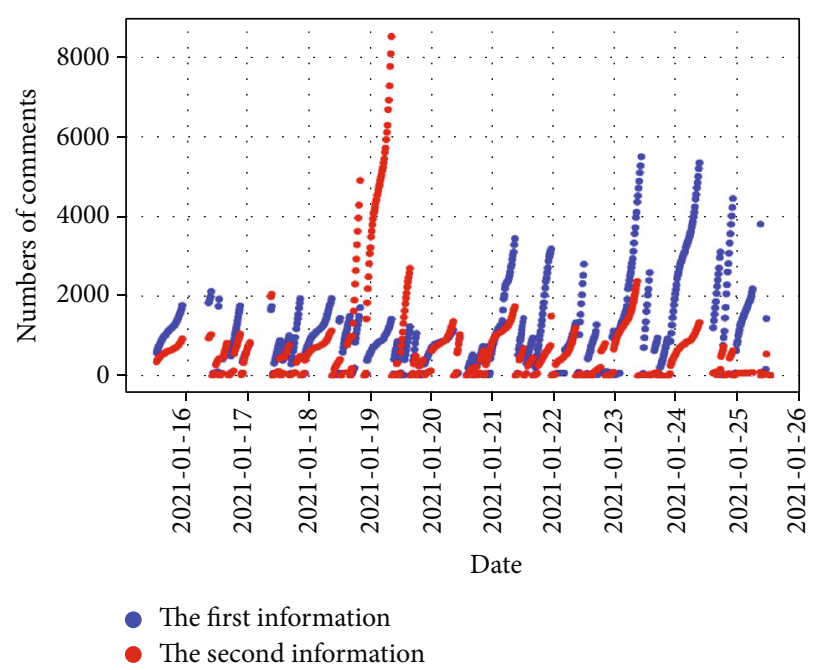

FIgURE 6: The information spreading effect of Toutiao.

series (Figure 6). Assuming that the average reading speed of users is constant, the effects of model parameters are discussed as follows.

(1) The visibility value of information $\beta$. As shown in Figure 6 , we can find that the dissemination effect of the first piece of information is better in most cases because the first information is for all users, and the second message is for different users. Therefore, the first piece of information has a higher visibility value. This phenomenon shows that the high visibility value promotes the information spreading on the news platform

(2) The quantity value of information $\alpha$. Although the first piece of information has a higher visibility value, the second piece of information sometimes spreads better. The main reason is that sometimes, users are more concerned with the second piece of information. Therefore, the quality of information is an important factor affecting information dissemination

(3) As shown in Figure 6, the influence of time on information dissemination is not obvious, mainly because the two pieces of information have high visibility value. Therefore, the news platform can enhance the visibility of specific topic information to maintain its dissemination effect

\section{Conclusions}

Based on the analysis of dynamic characteristics of the news platform, the paper models the dynamic characteristics of the user state and the value of information. Simulation experiments have been done to analyze the impact of dynamic characteristics on information spreading. Additionally, some applications are proposed and discussed. The main works of the paper are following: 
(1) The dynamic characteristics of information spreading on news platforms have been introduced. It is proposed that the dynamic of the user state and information value are involved in the process of information spreading

(2) We model the dynamic characteristics of the user state and information value and provide a detailed calculation process

(3) The related parameters of the dynamic model are assumed, and simulation experiments have been achieved under different parameters and conditions. The experimental results are analyzed in detail

(4) Based on the dynamic characteristics and the dynamic model, some applications are presented. Meanwhile, we analyzed the effect of its information dissemination by taking Toutiao as an example and confirmed that the visibility and quality of information are important factors that affect information spreading

Although this paper uses differential equations to construct a dynamic model of information spreading on news platforms, there are still some limitations. Firstly, we make some assumptions about some critical parameters of the model, such as the average reading speed $1 / r$, the value of information quantity $\alpha$, and information visibility $\beta$. How to determine these parameters is for further study. Secondly, a large amount of data is needed to verify and optimize the model, which is the goal of our future work.

\section{Data Availability}

The data used to support the findings of this study are available from the corresponding author upon request.

\section{Conflicts of Interest}

The authors declare no conflict of interest.

\section{Acknowledgments}

This work was supported by the National Key R\&D Program of China (No. 2019YFB1406201).

\section{References}

[1] "Number of monthly active users (MAU) of the leading news apps in China in December 2020," 2020, https://www.Statista .Com/Statistics/910787/China-Monthly-Active-Users-onLeading-News-Apps.

[2] E. Mussumeci and F. C. Coelho, "Reconstructing news spread networks and studying its dynamics," Social Network Analysis and Mining, vol. 8, no. 1, pp. 1-8, 2018.

[3] Y. Zhang, Y. Su, W. Li, and H. Liu, "Rumor and authoritative information propagation model considering super spreading in complex social networks," Physica A: Statistical Mechanics and its Applications, vol. 506, pp. 395-411, 2018.

[4] Y. Xiao, D. Chen, S. Wei, Q. Li, H. Wang, and M. Xu, "Rumor propagation dynamic model based on evolutionary game and anti-rumor," Nonlinear Dynamics, vol. 95, no. 1, pp. 523539, 2019.

[5] M. Jiang, Q. Gao, and J. Zhuang, "Reciprocal spreading and debunking processes of online misinformation: a new rumor spreading-debunking model with a case study," Physica A: Statistical Mechanics and its Applications, vol. 565, article 125572, 2021.

[6] K. Shu, D. Mahudeswaran, S. Wang, D. Lee, and H. Liu, "Fakenewsnet: a data repository with news content, social context, and spatiotemporal information for studying fake news on social media," Big Data, vol. 8, no. 3, pp. 171-188, 2020.

[7] O. N. Berduygina, T. N. Vladimirova, and E. V. Chernyaeva, "Trends in the spread of fake news in mass media," Media Watch, vol. 10, no. 1, pp. 122-132, 2019.

[8] D. Yang, T. W. S. Chow, L. Zhong, Z. Tian, and Q. Zhang, "True and fake information spreading over the Facebook," Physica A: Statistical Mechanics and its Applications, vol. 505, pp. 984-994, 2018.

[9] S. Jiang and H. Fan, "Credit risk contagion coupling with sentiment contagion," Physica A: Statistical Mechanics and its Applications, vol. 512, pp. 186-202, 2018.

[10] J. Li and C. Bin, "Modeling for information spreading basing on dynamics," Concurrency and Computation: Practice and Experience, vol. 31, no. 9, 2019.

[11] L. Gao, W. Wang, L. Pan, M. Tang, and H. F. Zhang, "Effective information spreading based on local information in correlated networks," Scientific Reports, vol. 6, no. 1, pp. 1-10, 2016.

[12] X. Liu, D. He, and C. Liu, "Information diffusion nonlinear dynamics modeling and evolution analysis in online social network based on emergency events," IEEE Transactions on Computational Social Systems, vol. 6, no. 1, pp. 8-19, 2019.

[13] Z. K. Zhang, C. Liu, X. X. Zhan, X. Lu, C. X. Zhang, and Y. C. Zhang, "Dynamics of information diffusion and its applications on complex networks," Physics Reports, vol. 651, pp. 134, 2016.

[14] Y. Wang, J. Wang, H. Wang, R. Zhang, and M. Li, “Users' mobility enhances information diffusion in online social networks," Information Sciences, vol. 546, pp. 329-348, 2021.

[15] Y. Wu, M. Li, J. Xiao, M. A. Orgun, and L. Xue, "The impact of discrimination on the spread of infectious diseases in complex networks," New Generation Computing, vol. 32, no. 3-4, pp. 193-211, 2014.

[16] Y. Li, H. V. Zhao, and Y. Chen, "An epidemic model for correlated information diffusion in crowd intelligence networks," International Journal of Crowd Science, vol. 3, no. 2, pp. 168183, 2019.

[17] R. Narang, S. Sarin, P. Singh, and R. Goyal, "Impact of reciprocity in information spreading using epidemic model variants," Information, vol. 9, no. 6, p. 136, 2018.

[18] B. Xu and L. Liu, "Information diffusion through online social networks," in 2010 IEEE International Conference on Emergency Management and Management Sciences, pp. 53-56, Beijing, China, 2010.

[19] V. D. Blondel, J. L. Guillaume, R. Lambiotte, and E. Lefebvre, "Fast unfolding of communities in large networks," Journal of Statistical Mechanics: Theory and Experiment, vol. 2008, no. 10, p. 10008, 2008.

[20] G. X. Sun and S. Bin, "A new opinion leaders detecting algorithm in multi-relationship online social networks," Multimedia Tools and Applications, vol. 77, no. 4, pp. 4295-4307, 2018. 
[21] H. Gong, C. Guo, and Y. Liu, "Measuring network rationality and simulating information diffusion based on network structure," Physica A: Statistical Mechanics and its Applications, vol. 564, article 125501, 2021.

[22] M. Yan, S. Li, C. A. Chan, Y. Shen, and Y. Yu, "Mobility prediction using a weighted Markov model based on mobile user classification," Sensors, vol. 21, no. 5, p. 1740, 2021.

[23] M. Yan, H. Yuan, Z. Li, Q. Lin, and J. Li, "Energy savings of wireless communication networks based on mobile user environmental prediction," Journal of Environmental Protection and Ecology, vol. 22, no. 1, pp. 206-217, 2021.

[24] H. Lu, M. Zhang, and S. Ma, "Between clicks and satisfaction: study on multi-phase user preferences and satisfaction for online news reading," in The 41st International ACM SIGIR Conference on Research \& Development in Information Retrieval, pp. 435-444, Ann Arbor, MI, USA, 2018.

[25] C. Wu, F. Wu, T. Qi, and Y. Huang, "User modeling with click preference and reading satisfaction for news recommendation," in International Joint Conference on Artificial Intelligence, pp. 3023-3029, Yokohama, Japan, 2020.

[26] S. Wallace, R. Treitman, N. Kumawat, and K. Arpin, “Towards readability individuation: the right changes to text format make large impacts on reading speed," Journal of Vision, vol. 20, no. 10, pp. 17-17, 2020.

[27] B. Shaver-Troup, K. Crowley, and M. Jordan, “Optimizing reading performance by manipulating the shape, size, and spacing of text to match the individual's visual processing capacity," Collective Impact Project, rev Reading, 2017. 